\title{
Restoration of wild-type virulence to Tri5 disruption mutants of Gibberella zeae via gene reversion and mutant complementation
}

\author{
Robert H. Proctor, ${ }^{1}$ Thomas M. Hohn ${ }^{2}$ and Susan P. McCormick ${ }^{2}$ \\ Author for correspondence: Robert H. Proctor. Tel: +1 309681 6216. Fax: +1 3096816686. \\ e-mail: proctorh@mail.ncaur.usda.gov
}

1 Bioactive Agents Unit, National Center for Agricultural Utilization Research, United States Department of

Agriculture, Agricultural Research Service, Peoria, IL 61604, USA

2 Mycotoxin Research Unit, National Center for Agricultural Utilization Research, United States Department of Agriculture, Agricultural Research Service, Peoria, IL 61604, USA

\begin{abstract}
Gibberella zeae is a pathogen of small grain crops and produces trichothecene mycotoxins in infected host tissue. The role of trichothecenes in the virulence of G. zeae was previously investigated using trichothecene-non-producing mutants that were generated via transformation-mediated disruption of a gene (Tri5) that encodes the first enzyme in the trichothecene biosynthetic pathway. The mutants were less virulent on some hosts than the wild-type strain from which they were derived. Here, we used two approaches to determine whether the reduced virulence of mutants was due specifically to Tri5 disruption or to non-target effects caused by the transformation process. First, we generated a revertant from a Tri5 disruption mutant by allowing the mutant to pass through the sexual phase of its life cycle. In approximately $2 \%$ of the resulting progeny the disrupted Tri5 had reverted to wild-type; however, only one of three revertant progeny also regained the ability to produce trichothecenes. In the second approach, we complemented the Tri5 mutation in a disruption mutant by transforming the mutant with a plasmid carrying a functional copy of Tri5. In all transformants examined, the ability to produce trichothecenes was restored. The restoration of trichothecene production in the revertant progeny and in the complemented mutant was accompanied by restoration of wild-type or near wild-type levels of virulence on wheat seedlings (cultivar Wheaton). The results indicate that the reduced virulence of the mutants was caused by disruption of Tri5 rather than nontarget effects resulting from the transformation process. The results also provide further evidence that trichothecenes contribute to the virulence of plant-pathogenic fungi.
\end{abstract}

Keywords: Gibberella zeae, trichothecene, Tri5, gene disruption

\section{INTRODUCTION}

Gibberella zeae (Schwein.) Petch (anamorph Fusarium graminearum) is a filamentous ascomycete that causes severe and economically important diseases on a variety of plants, including maize, barley, wheat and carnation (Nelson et al., 1975; Cook, 1981; Kommedahl \& Windels, 1981). On small grains, G. zeae can cause seedling blight, root rot and head blight (or scab). In addition, G. zeae produces the trichothecene mycotoxins deoxynivalenol and acetylated derivatives of deoxynivalenol in infected grain (Cook, 1981; Atanassov et al., 1994). Trichothecenes are sesquiterpenoids produced by several fungal genera, including species of Fusarium, Myrothecium, Stachybotrys and Trichothecium, and by two species of the plant genus Baccharis
(Sharma \& Kim, 1991; Jarvis et al., 1991). These toxins are harmful to both plants and animals and their toxicity is thought to occur as a result of their ability to inhibit protein biosynthesis (Sharma \& Kim, 1991; Wang \& Miller, 1988; Jarvis, 1991). The phytotoxicity of trichothecenes and the fact that many trichothecene-producing fungi are plant pathogens has lead to the hypothesis that these toxins are virulence factors. Indeed, mutants of $F$. sporotrichioides, G. pulicaris and G. zeae that are unable to produce trichothecenes exhibit reduced virulence on some hosts (Desjardins et al., 1989, 1992; Proctor et al., 1995). The trichothecene-deficient mutants of $G$. pulicaris and G. zeae were generated via transformationmediated disruption of a gene, Tri5, that encodes the enzyme trichodiene synthase. This enzyme catalyses the cyclization of the isoprenoid intermediate farnesyl 
pyrophosphate to trichodiene, which serves as the parent compound for all trichothecenes (Hohn \& VanMiddlesworth, 1986). The formation of trichodiene marks the biochemical step at which trichothecene biosynthesis branches from general isoprenoid biosynthesis. Disruption of Tri5 prevents the formation of functional trichodiene synthase and as a result precludes the formation of trichothecenes or intermediates in trichothecene biosynthesis. However, disruption of Tri5 should not prevent the formation of isoprenoids for which trichodiene is not an intermediate.

Transformation-mediated gene disruption is a powerful tool for examining the role of genes in the pathogenicity of fungi on plants (Desjardins et al., 1992; Herrmann et al., 1996; Bowyer et al., 1995; Panaccione et al., 1992; Rogers et al., 1994; Stahl et al., 1994; VanEtten et al., 1995). Gene disruption has the advantage over chemical and UV light-induced mutagenesis in that it allows for the generation of defined mutations in targeted genes. However, the transformation process itself can cause alterations at loci in addition to the targeted gene (Keller et al., 1990; Oliver \& Osbourn, 1995). Thus, a change in virulence that accompanies gene disruption is not always sufficient proof that the target gene is involved in pathogenicity. Several approaches have been employed to determine whether observed reductions in virulence following gene disruption are caused by the disruption or by non-target effects of transformation. These include (i) analysis of more than one disruption mutant (Desjardins et al., 1992; Herrmann et al., 1996; Talbot et al., 1996), (ii) analysis of transformants in which the disruption vector integrated ectopically (Desjardins $e t$ al., 1992; Herrmann et al., 1996; Talbot et al., 1996), (iii) complementation of the disruption mutant with a wild-type copy of the disrupted gene via a second transformation (Talbot et al., 1996) and (iv) outcrossing a disruption mutant with a wild-type strain and analysing the resulting meiotic progeny for cosegregation of the disrupted gene and reduced virulence (Desjardins $e t$ al., 1992; Talbot et al., 1996). In the initial characterization of trichothecene-non-producing mutants of G. zeae, the virulence of two Tri5 disruption mutants and a transformant in which the disruption vector had integrated at an ectopic site was examined (Proctor et al., 1995). The other approaches were not employed because a suitable second selectable marker was not available and although G. zeae can outcross, analysis of the resulting meiotic progeny is cumbersome because the fungus is homothallic.

In this study, we used two approaches to determine whether the reduced virulence of Tri5 mutants of $G$. zeae was due specifically to disruption of this gene or to non-target effects resulting from the transformation process. The first approach was based on the observation that in filamentous fungi closely linked duplicated sequences can recombine when the fungi pass through the sexual phase of their life cycle and as a result of this recombination one copy of the duplicated sequence can be deleted in meiotic progeny (Desjardins et al., 1992; Miller et al., 1986; Selker et al., 1987). We exploited this phenomenon to generate a trichotheceneproducing, meiotic progeny with a wild-type Tri5 from a disruption mutant that had two closely linked, mutated copies of the Tri5 coding region. In the second approach, we complemented the Tri5 mutation in a disruption mutant by introducing a functional copy of Tris into the mutant via a second transformation.

\section{METHODS}

Fungal strains and manipulations. The trichothecene-producing, wild-type strain, GZ3639 (previously Z3639) and the trichothecene-deficient, Tri5 disruption mutants GZT33 and GZT40 have been described previously (Proctor et al., 1995). These strains were routinely cultured on V8 juice agar (Tuite, 1969) at $25^{\circ} \mathrm{C}$ under white and blue fluorescent lights for $12 \mathrm{~h}$ $\mathrm{d}^{-1}$. GZT33 was selfed following a method similar to that described to cross G. fujikuroi (Klittich \& Leslie, 1988). GZT33 was grown on carrot agar at $25^{\circ} \mathrm{C}$ under fluorescent light as described above for 5-7 d. At this time, $2 \mathrm{ml}$ sterile water was added to the culture and spread with a glass rod to flatten the mycelium. Excess water was then removed and the cultures were incubated for an additional 1-2 weeks until cirrhi were observed oozing from developing perithecia. To obtain single ascospore cultures, a single cirrhus was picked and vortexed vigorously in $1 \mathrm{ml}$ water. The resulting suspension was then spread over $3 \%(\mathrm{w} / \mathrm{v})$ water agar and incubated for 8-10 h. With the aid of a stereomicroscope, small blocks of agar around individual germinating ascospores were cut from the water agar and transferred to V8 juice agar. Care was taken to avoid clumps of two or more ascospores.

Nucleic acid manipulations. Except when indicated otherwise, fungal genomic DNA was isolated as described previously (Hohn \& Desjardins, 1992) except that after the chloroform extraction, the aqueous phase was incubated with RNase for $30 \mathrm{~min}$ at room temperature, combined with an equal volume of $5 \mathrm{M} \mathrm{LiCl}$, incubated for $15 \mathrm{~min}$ on ice and then centrifuged at $14000 \mathrm{~g}$ for $10 \mathrm{~min}$. DNA was precipitated from the supernatant with 2.5 vols $95 \%(\mathrm{v} / \mathrm{v})$ ethanol and resuspended in Tris/EDTA buffer, pH 8 (Sambrook et al., 1989). Southern blotting and PCR experiments were as described previously except for the modifications noted below (Hohn \& Desjardins, 1992; Proctor et al., 1995).

In PCR experiments designed to distinguish between the disrupted and wild-type Tri5, DNA templates were obtained by growing individual strains on V8 juice agar in $100 \times 15 \mathrm{~mm}$ Petri plates until the mycelium covered the entire agar surface. At this time, the mycelium was scraped from the agar and dried under vacuum overnight. The mycelium was then ground, mixed with extraction buffer and extracted with phenol/chloroform $(1: 1)$. DNA was purified from the aqueous phase using GeneClean (Bio 101) as described by the manufacturer except that samples were rinsed only once with NEW solution and DNA was eluted with $20 \mu \mathrm{l}$ of Tris/EDTA buffer (Sambrook et al., 1989). Two to five microlitres of the resulting solution was used as a template in PCR. The sequences of the PCR primers were as follows: $65,5^{\prime}$ GAAACAGCTATGACCATG $3^{\prime} ; 170,5^{\prime}$ GGCATGGTTGTATACAGC 3'; 246, 5' CTCCTTCACATCGTCCTTGG 3'; 248, 5' CTATGCCTACAGCATCCAGG 3'.

Construction of Tri5 complementation plasmid. A plasmid containing a functional Tri5 gene, consisting of the $5^{\prime}$ flanking and coding regions of the G. zeae Tri5 and the $3^{\prime}$ flanking region of the $F$. sporotrichioides Tri5, was constructed to 
(a)

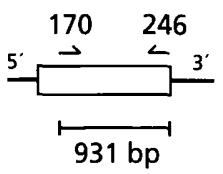

(b)
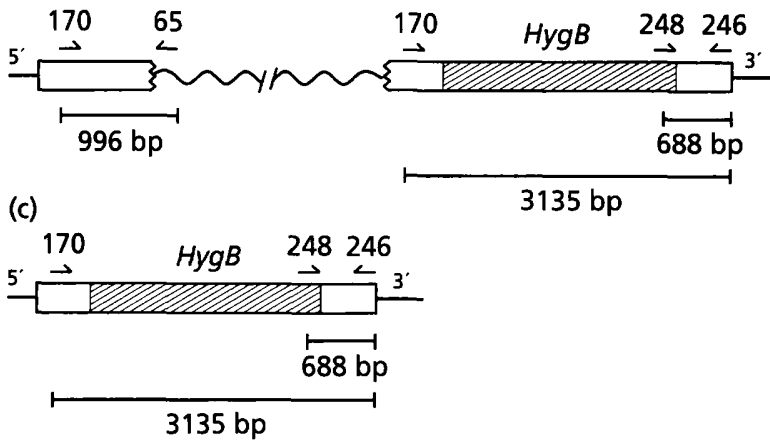

Fig. 1. Maps of Tri5 alleles and $P C R$ strategy for distinguishing between them in progeny from disruption mutant GZT33. (a)Tri5-1 allele in wild-type strain GZ3639; (b)Tri5-2 (disrupted) allele in GZT33; (c) Tri5-3 (disrupted) allele in GZT40. Open boxes represent the Tri5 coding region, jagged ends of open boxes represent truncated ends of the coding region, hatched boxes represent the $H y g B$ marker and the wavy line represents the bacterial cloning vector sequence. The numbered arrows indicate positions of sequences complementary to PCR primers $65,170,246$ and 248 and the direction of the arrows indicate the direction in which DNA synthesis is primed. Bars indicate sizes of possible PCR products.

facilitate the complementation of Tri5 disruption mutant GZT40. The $5^{\prime}$ flanking sequences were isolated by rescuing a portion of plasmid pGZTS4-1 from transformant GZT33 (Proctor et al., 1995). Plasmid rescue was accomplished by digesting genomic DNA from GZT33 with Sall. Following Sall digestion, the DNA fragments were isolated using GeneClean and then ligated to themselves. Transformation of Escherichia coli with the resulting ligation products yielded eight colonies. Analysis of six of these revealed that three carried the expected rescued plasmid (pRESC1-4) while the other three carried the intact disruption plasmid pGZTS4-1 (Proctor et al., 1995). The plasmid pRESC1-4 was found to contain $776 \mathrm{bp}$ of sequence upstream of the Tri5 coding region. Construction of the complementation plasmid began with a $281 \mathrm{bp}$ fragment amplified from the $3^{\prime}$ flanking sequence of the F. sporotrichioides Tri5 gene (nt 3-284 downstream of the stop codon). The primers used to amplify this fragment contained Bst XI and NotI sites and the resulting product was cloned into pBluescriptII KS(-) to yield pADD1-3. The SalI-HindIII fragment from pRESC4-1 containing the $776 \mathrm{bp}$ promoter region and nt $1-829$ of the G. zeae Tri5 coding region was then cloned into pADD1-3 to generate pADD2B. Next, an amplified portion of the G. zeae Tri5 coding region was cloned into Xhol/NotI-cut pADD2B to yield pADD3. This was accomplished using the existing Xhol site at nt 49 of the G. zeae Tri5 and a NotI site introduced via the PCR primers immediately $3^{\prime}$ of the Tri5 stop codon. The entire chimeric Tri5 gene in pADD3 was amplified and cloned in the TA cloning vector pCRII (Invitrogen) to yield pADD4. The complementation plasmid pADD6-1 was then constructed by transferring the chimeric Tri5 gene from pADD4 as an $A p a \mathrm{I}-K p n \mathrm{I}$ fragment into the fungal transformation vector

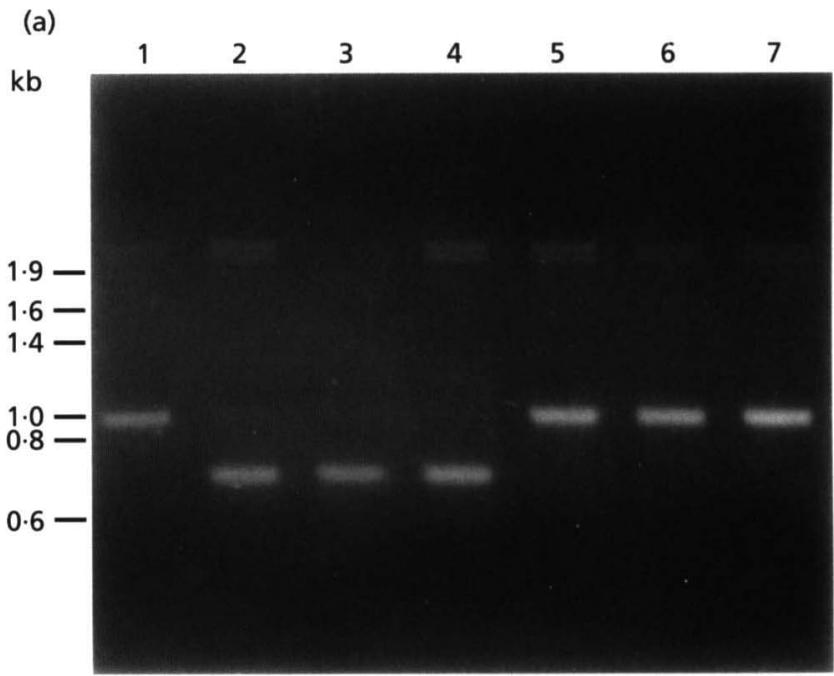

(b)

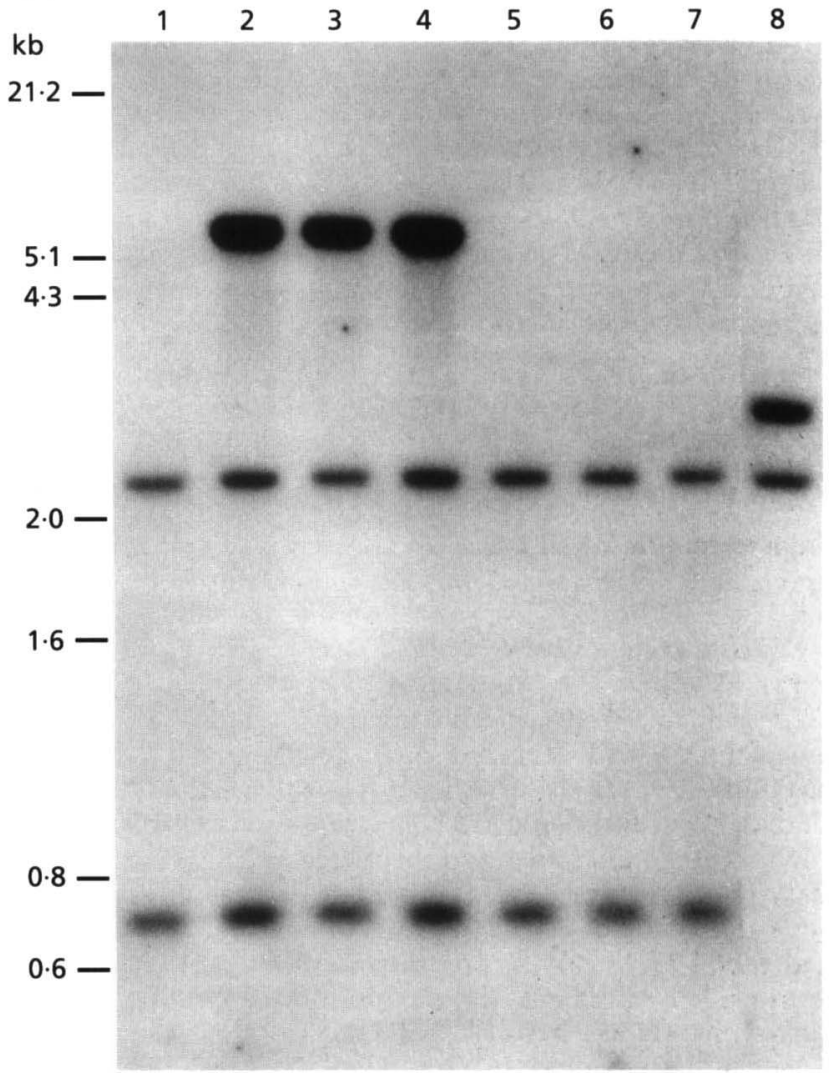

Fig. 2. (a) PCR analysis of selected GZT33 progeny for wild-type Tri5. Bands are the amplification products from reactions with genomic DNA templates isolated from wild-type strain GZ3639 (lane 1), Tri5 mutant parent GZT33 (lane 2) and progeny GZP1-1 (lane 3), GZP3-1 (lane 4), GZP1-6 (lane 5), GZP1-15 (lane 6) and GZP3-9 (lane 7). (b) Southern blot analysis of GZT33 progeny. Genomic DNA was digested with $X m n l$ and probed with ${ }^{32} \mathrm{P}$ labelled wild-type Tri5 coding region (nt 2-1187). Lanes: 1, GZ3639; 2, GZT33; 3, GZP1-1; 4 GZP3-1; 5, GZP1-6; 6, GZP1-15; 7, GZP3-9; 8, GZP5-15. 
Table 1. Trichothecene production and virulence of progeny of Tri5 disruption mutant GZT33

\begin{tabular}{|c|c|c|c|c|}
\hline Strain & Genotype $^{*}$ & $\begin{array}{c}\text { Trichothecene } \\
\text { production } \dagger\end{array}$ & $\begin{array}{c}\text { Emergence } \\
(\%) \ddagger\end{array}$ & $\begin{array}{c}\text { Seedling } \\
\text { height } \\
(\mathrm{cm}) \neq\end{array}$ \\
\hline Control (water) & - & - & $92 \cdot 7$ a & $26 \cdot 2$ a \\
\hline GZT33 & Tri5-2 & 0 & $53.3 \mathrm{c}$ & $27 \cdot 7 \mathrm{ab}$ \\
\hline GZP1-1 & Tri5-2 & 0 & $56.7 \mathrm{bc}$ & $27 \cdot 0 \mathrm{ab}$ \\
\hline GZP1-4 & Tri5-2 & 0 & $67 \cdot 3 \mathrm{~b}$ & $28 \cdot 4 \mathrm{~b}$ \\
\hline GZP3-1 & Tri5-2 & 0 & $58.7 \mathrm{bc}$ & $28 \cdot 0 \mathrm{ab}$ \\
\hline GZP1-6 & Tri5-1 & 0 & $62.0 \mathrm{bc}$ & $27 \cdot 3 \mathrm{ab}$ \\
\hline GZP1-15 & Tri5-1 & 0 & $62.7 \mathrm{bc}$ & $26.8 \mathrm{ab}$ \\
\hline GZP3-9 & Tri5-1 & 238 & $25 \cdot 3 \mathrm{~d}$ & $8 \cdot 2 c$ \\
\hline GZ3639 & Tri5-1 & 209 & $29 \cdot 0 \mathrm{~d}$ & $8.9 \mathrm{c}$ \\
\hline
\end{tabular}

* Genotypes were determined by PCR and Southern blot analysis (Fig. 2). Tri5-1 is the wild-type Tri5 allele. Tri5-2 is the disrupted Tri5 allele present in GZT33.

† Data are expressed as $\mu \mathrm{g}$ triacetyldeoxynivalenol ( $\mathrm{g}$ dry wt corn meal culture $)^{-1}$ and are the means from two or more assays.

$\ddagger$ Data are means from three experiments. In each experiment there were five pots per treatment (strain) and 10 seeds inoculated with $G$. zeae or water were sown in each pot. Emergence is the percentage of seedlings that emerged from the 10 seeds that were sown. Seedling height was determined by measuring the length of seedlings from the seed to the tip of the tallest leaf. Seedlings that did not emerge were not included in analysis of seedling height data. The overall $F$-tests for strains were significant $(P<0 \cdot 01)$. For percentage emergence, the mean square error (variance for the entire data set) was 20.3 with 117 degrees of freedom. For seedling height, the mean square error was 56.3 with 694 degrees of freedom. Within a column, means not followed by the same letter are significantly different $(P<0.05)$ according to a paired $t$-test of least squares means.

pAMDS4-1 (J. Jones, T. Leathers \& T. Hohn, unpublished) which contains the Aspergillus nidulans amdS gene.

Transformation of GZT40. Protoplasting and transformation procedures were carried out at room temperature unless otherwise indicated and were similar to those described previously (Hohn \& Desjardins, 1992; Salch \& Beremand, 1993). To prepare protoplasts, conidia of GZT40 were harvested from 7-10-d-old V8 juice agar cultures and used to inoculate $250 \mathrm{ml}$ Erlenmeyer flasks containing $100 \mathrm{ml} \mathrm{YEPD}$ medium (w/v, $1 \%$ peptone, $0.3 \%$ yeast extract, $2 \%$ glucose). Conidia were inoculated at a concentration of between $1 \times 10^{6}$ and $5 \times 10^{6}$ conidia $\mathrm{ml}^{-1}$ and incubated at $28^{\circ} \mathrm{C}$ for $8-9 \mathrm{~h}$ at 200 r.p.m. Germlings were harvested by centrifugation in a table top centrifuge at $3000 \mathrm{~g}$ for $10 \mathrm{~min}$ and washed twice with $50 \mathrm{ml} 0.7 \mathrm{M} \mathrm{NaCl}$. After the second wash, the pellet was resuspended in $20 \mathrm{ml}$ of a solution containing $100 \mathrm{mg}$ Novozyme (Novo Nordisk), $500 \mathrm{mg}$ driselase (Sigma) and $1 \mathrm{mg}$ chitinase (Sigma). Incubation at $30^{\circ} \mathrm{C}$ resulted in $100 \%$ protoplast formation within $30-90 \mathrm{~min}$. Protoplasts were pelleted at $3000 \mathrm{~g}$ for $5 \mathrm{~min}$ and resuspended in $10 \mathrm{ml} 0.7 \mathrm{M}$ $\mathrm{NaCl}$. Protoplasts were again pelleted at $3000 \mathrm{~g}$ for $5 \mathrm{~min}$ and washed twice in $10 \mathrm{ml} \mathrm{STC}(0.8 \mathrm{M}$ sorbitol, $0.05 \mathrm{M}$ Tris, $\mathrm{pH}$ $\left.8.0,50 \mathrm{mM} \mathrm{CaCl}_{2}\right)$. After the second wash, the protoplast pellet was resuspended in STC to $1 \times 10^{8}$ protoplasts $\mathrm{ml}^{-1}$ and then further diluted with SPTC (STC with $40 \%$, w/v, PEG 6000 ) and DMSO (protoplasts/SPTC/DMSO, 8:2:0:1). The resulting solution was frozen as $100 \mu \mathrm{l}$ aliquots at $-80^{\circ} \mathrm{C}$.

Transformation was performed by thawing a frozen $100 \mu \mathrm{l}$ protoplast aliquot on ice and diluting with $100 \mu \mathrm{l}$ STC containing $1-10 \mu \mathrm{g}$ DNA and $50 \mu \mathrm{l} 30 \%$ PEG. Following a $20 \mathrm{~min}$ incubation at room temperature, an additional $2 \mathrm{ml}$ of
$30 \%$ PEG was carefully added and incubation was continued at room temperature for $5 \mathrm{~min}$. The transformation mixture was diluted further with $4 \mathrm{ml} \mathrm{STC}$ and then $600 \mu \mathrm{l}$ aliquots were mixed with $6.5 \mathrm{ml}$ molten SL medium containing $15 \mathrm{mM}$ $\mathrm{CsCl}$ and $1 \%$ agarose and spread on SL medium. SL medium consisted of a previously described basal medium (Correll et al., 1987) supplemented with $10 \mathrm{mM}$ acetamide (Sigma). Transformation plates were incubated at $28^{\circ} \mathrm{C}$ and transformants were initially detected after 2 or $3 \mathrm{~d}$. Putative transformants were transferred to SL agarose slants prior to picking single spore isolates.

Trichothecene analysis. The ability of $G$. zeae to produce trichothecenes was analysed by culturing the fungus on sterile corn meal, extracting the cultures with ethyl acetate and analysing the extract via GC and GC-MS as described previously (Proctor et al., 1995).

Virulence assays. Virulence assays were carried out, as described previously (Proctor et al., 1995), by soaking wheat seeds of cultivar Wheaton in a suspension of $5 \times 10^{5}$ macroconidia $\mathrm{ml}^{-1}$ and then sowing the seeds into moistened vermiculite. Disease was assessed 2 weeks after sowing.

\section{RESULTS}

\section{Reversion of disrupted Tri5}

The Tri5 locus (allele Tri5-2) in disruption mutant GZT33 contains two copies of the Tri5 coding region (nt 2-1098) separated from one another by about $3 \mathrm{~kb}$ of bacterial vector sequences (Fig. 1b) (Proctor et al., 1995). In addition, the downstream copy of the Tri5 coding 
region in GZT33 is interrupted by the $H y g B$ gene. This Tri5 duplication made GZT33 a good candidate for gene reversion experiments because recombination between the duplicated sequences could restore the wildtype Tri5 allele (Tri5-1). We speculated that this sort of recombination might be induced by passing GZT33 through the sexual phase of its life cycle. Because G. zeae is homothallic, it was possible to self-fertilize GZT33 and obtain viable ascospore progeny. One hundred and forty of these progeny were isolated ( 20 from each of seven perithecia) and screened for the presence of the wild-type Tri5 using two PCR protocols. In the first protocol, a combination of three oligonucleotides (170, 246 and 248) were used as primers (Fig. 1). When these three oligonucleotides are combined in one reaction, oligonucleotides 170 and 246 amplify a 931 bp product from a wild-type Tri5 DNA template. However, if a disrupted Tri5 serves as the template, oligonucleotides 246 and 248 amplify a 688 bp product (Fig. 1). Oligonucleotides 170 and 246 can also amplify a 3135 bp product from a disrupted Tri5 template, but this product was generally not detected under our PCR conditions. In the second PCR protocol, oligonucleotides 65 and 170 were employed. These primers amplify a 996 bp product from a Tri5-2 template but fail to amplify a product from the wild-type Tri5 template (Fig. 1). Genomic DNA of selected progeny was also analysed by Southern blot analysis using XmnI-digested genomic DNA and the wild-type Tri5 coding region as a hybridization probe.

PCR analysis of the 140 progeny of GZT33 indicated that three progeny, GZP1-6, GZP1-15 and GZP3-9, carried a wild-type Tri5. In the PCR analysis in which oligonucleotides 170, 246 and 248 were employed, the $931 \mathrm{bp}$ fragment indicative of the wild-type Tri5 was amplified from these three progeny (Fig. 2a). No fragment was amplified from these progeny in PCR analysis employing oligonucleotides 65 and 170, which is also consistent with the presence of a wild-type Tri5 in these three progeny. In addition, Southern analysis of XmnI-digested DNA from progeny GZP1-6, GZP1-15 and GZP3-9 yielded a hybridization pattern identical to the pattern obtained with wild-type DNA (Fig. 2b). A fourth progeny, GZP5-15, appeared to have a disrupted Tri5 gene as indicated by the amplification of a $688 \mathrm{bp}$ fragment in the PCR analysis employing oligonucleotides 170, 246 and 248. However, in PCR with oligonucleotides 65 and 170, no fragment was amplified, suggesting that the structure of the disrupted Tri5 in GZP5-15 was different from the Tri5-2 allele present in GZT33. Southern analysis of XmnI-digested DNA from GZP5-15 revealed a hybridization pattern different from that obtained with both GZT33 and GZ3639 (wildtype) DNA (Fig. 2b). Comparison of the hybridization pattern with previously published hybridization patterns (Proctor et al., 1995) indicated that the structure of Tri5 in GZP5-15 is similar to that obtained with the Tri5 disruption mutant GZT40. PCR analyses of the remaining 136 progeny from GZT33 were consistent with the presence of the Tri5-2 (disrupted) allele in these

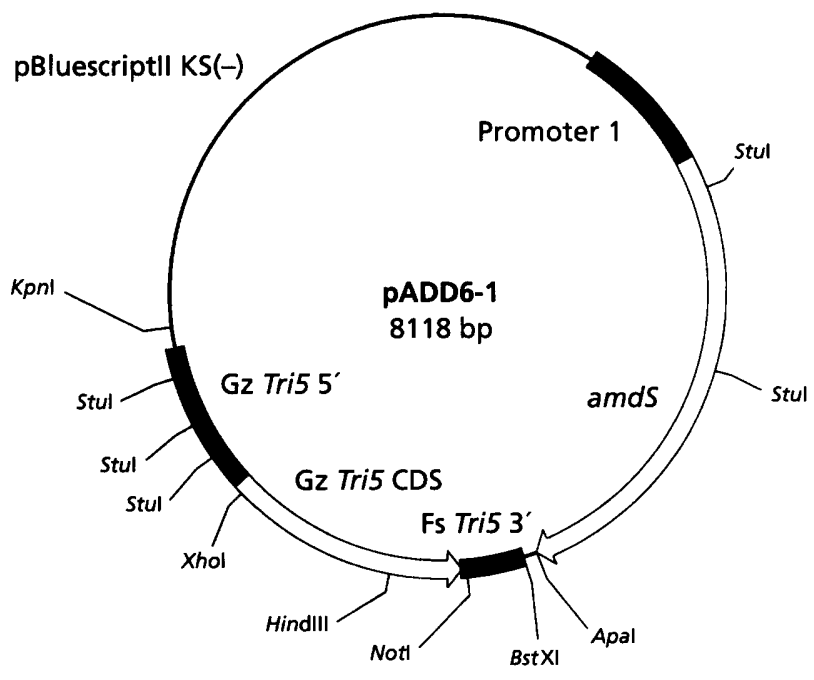

Fig. 3. Map of plasmid pADD6-1. Abbreviations: Gz Tri5 5', $5^{\prime}$ flanking sequence of $G$. zeae Tri5; Gz Tri5 CDS, coding region of $G$. zeae Tri5; Fs Tri5 3', 3' flanking sequence of F. sporotrichioides Tri5; amdS, coding region and 3' flanking sequence of amdS gene from $A$. nidulans; Promoter 1, Promoter 1 sequence from Cochliobolus heterostrophus. Only those restriction sites employed for cloning or Southern blot analysis are shown.

progeny. The PCR and Southern results from two representative progeny, GZP1-1 and GZP3-1, of this type are shown in Fig. 2.

Strains GZ3639 and GZT33 and selected progeny were analysed for their ability to produce trichothecenes. Parent strain GZT33 and progeny GZP1-1, GZP1-4 and GZP3-1, all of which carry a disrupted Tri5-2 allele, did not produce trichothecenes (Table 1). Surprisingly, putative revertant progeny GZP1-6 and GZP1-15 also failed to produce trichothecenes. The only progeny that produced trichothecenes was the Tri5 revertant GZP3-9 and it did so at levels that were comparable to those produced by the wild-type progenitor, GZ3639 (Table 1).

\section{Complementation of Tri5 disruption mutant}

The Tri5 disruption mutant GZT40 carries a single, non-functional copy of the Tri5 gene (allele Tri5-3) that is interrupted by the $\mathrm{HygB}$ gene (Fig. 1) (Proctor et al., 1995). To complement the disrupted Tri5 gene in GZT40 it was necessary to first construct complementation vector, pADD6-1 (Fig. 3), carrying a functional copy of Tri5 (allele Tri5-4). This functional Tri5 consisted of the $776 \mathrm{bp}$ promoter region and $1187 \mathrm{bp}$ coding region of the wild-type G. zeae Tri5 and a $281 \mathrm{bp}$ fragment containing the transcription termination sequence from the wild-type $F$. sporotrichioides Tri5. The vector pADD6-1 also carried the $A$. nidulans amdS gene (Corrick \& Hynes, 1987) fused to Promoter 1 from Cochliobolus beterostrophus (Turgeon et al., 1987). This chimeric Promoter 1/amdS gene facilitated the 
Table 2. Trichothecene production and virulence of the complemented Tri5 disruption mutant of G. zeae

\begin{tabular}{|c|c|c|c|c|}
\hline Strain & Genotype* & $\begin{array}{c}\text { Trichothecene } \\
\text { production } \dagger\end{array}$ & $\begin{array}{c}\text { Emergence } \\
(\%) \neq\end{array}$ & $\begin{array}{c}\text { Seedling } \\
\text { height } \\
(\mathrm{cm}) \neq\end{array}$ \\
\hline Control (water) & - & - & $93 \cdot 1$ a & $19 \cdot 4 \mathrm{~b}$ \\
\hline GZT40 & Tri5-3 & 0 & $68 \cdot 7 \mathrm{~b}$ & $21 \cdot 1 \mathrm{a}$ \\
\hline GZT201-1 & Tri5-3/Tri5-4 & 670 & $45 \cdot 0 \mathrm{c}$ & $18 \cdot 7 \mathrm{~b}$ \\
\hline GZT201-5 & Tri5-3/Tri5-4 & 434 & $31.3 \mathrm{~cd}$ & $19 \cdot 8 \mathrm{ab}$ \\
\hline GZ3639 & Tri5-1 & 224 & $22 \cdot 5 \mathrm{~d}$ & $19 \cdot 5 \mathrm{ab}$ \\
\hline
\end{tabular}

*Genotypes were determined by PCR and Southern blot analysis (Fig. 4). Tri5-1 is the wild-type Tri5 allele. Tri5-3 is the disrupted allele present in GZT40. Tri5-4 is a functional copy of Tri5 derived from G. zeae and F. sporotrichioides sequences (see Methods).

† Data are expressed as $\mu \mathrm{g}$ triacetyldeoxynivalenol ( $\mathrm{g}$ dry wt corn meal culture $)^{-1}$ and are the means from two assays.

$\ddagger$ Data are means from two experiments. Virulence assays were designed and the data analysed as described for Table 1 except that there were eight pots per treatment in each experiment. The overall $F$-tests for strains were significant $(P<0 \cdot 01)$. For percentage emergence, the mean square error (variance for the entire data set) was 20.7 with 70 degrees of freedom. For seedling height, the mean square error was 16.8 with 409 degrees of freedom. Within a column, means not followed by the same letter are significantly different $(P<0.01)$ according to a paired $t$-test of least squares means.

selection of pADD6-1 transformants by their ability to grow on media in which acetamide is the sole source of nitrogen.

Transformation of GZT40 with pADD6-1 resulted in the isolation of four transformants. Two transformants, GZT201-1 and GZT201-5, were selected for further analysis. Single spore isolates of these transformants were found to be restored to trichothecene production when grown on corn meal (Table 2). Southern blot analysis revealed that the integration of pADD6-1 had occurred ectopically in GZT201-1 but at the Tri5 locus in GZT201-5 (Fig. 4).

\section{Virulence of Tri5 revertant}

Virulence of G. zeae strains was evaluated via a seedling blight assay (Proctor et al., 1995). In the assay, disease severity was assessed by measuring the ability of the fungus to inhibit seedling emergence and the growth of seedlings once they had emerged. In virulence assays for the reversion study, the results obtained by measuring emergence and growth (i.e. seedling height) showed the same trend. The Tri5 disruption mutant GZT33 caused significantly less $(P<0.05)$ seedling blight than its wildtype progenitor, GZ3639 (Table 1). Five, trichothecenedeficient progeny of GZT33, including three with a disrupted Tri5 (GZP1-1, GZP1-4 and GZP3-1) and the two with a revertant Tri5, (GZP1-6 and GZP1-15), also caused less disease than GZ3639. With the exception of GZP1-4, these progeny did not differ significantly from GZT33 in their ability to cause seedling blight (Table 1). In marked contrast to its siblings, the trichotheceneproducing, Tri5 revertant progeny, GZP3-9, caused significantly more seedling blight than GZT33 (Table 1). In addition, the severity of seedling blight caused by progeny GZP3-9 did not differ significantly from that caused by the wild-type progenitor, GZ3639 (Table 1). Thus, the trichothecene-deficient strains caused consistently less disease than the trichothecene-producing strains.

\section{Virulence of complemented Tri5 disruption mutant}

We examined the virulence of transformants GZT201-1 and GZT201-5 in the wheat seedling blight assay. In contrast to previous experiments (Proctor et al., 1995), seedling height was not a reliable indicator of differences in the virulence of the Tri5 disruption mutant GZT40 and the wild-type progenitor, GZ3639. In the first virulence assay carried out for this study, the height of seedlings from water- or GZT40-inoculated seeds was significantly greater $(P<0.05)$ than that of seedlings derived from seeds inoculated with GZ3639, GZT201-1 or GZT201-5. However, in the second assay, there were no significant differences in the height of seedlings from any G. zeae inoculation treatments. On the other hand, emergence data were consistent in both experiments. The wild-type progenitor caused significantly greater $(P<0.01)$ inhibition of seedling emergence than the Tri5 mutant GZT40 in both assays. In addition, the two complemented Tri5 mutants, GZT201-1 and GZT2015 , caused greater inhibition of seedling emergence than GZT40. Statistical analysis of emergence data also indicated that GZT201-1 caused significantly less $(P<0.01)$ inhibition of emergence than the wild-type progenitor strain GZ3639 (Table 2).

\section{DISCUSSION}

Transformation-mediated gene disruption has been used increasingly in recent years to evaluate the role of fungal genes in pathogenicity (Herrmann et al., 1996; Bowyer 


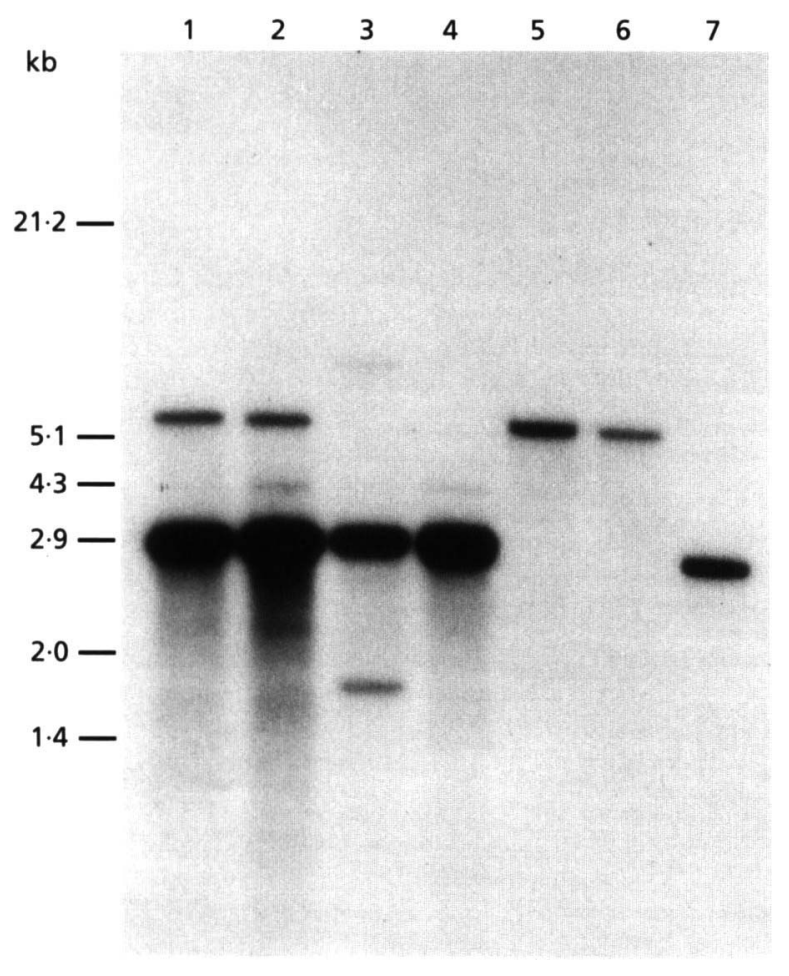

Fig. 4. Southern blot analysis of Stul-digested genomic DNA from pADD6-1 transformants. Blots were probed with ${ }^{32} \mathrm{P}$ labelled G. zeae Tri5 coding region. Lanes: 1, GZT201-1; 2, GZT201-2; 3, GZT201-3; 4, GZT201-5; 5, GZT201-6; 6, GZT40; 7, GZ3639 (wild-type). Only transformants GZT201-1, GZT201-2, GZT201-3 and GZT201-5 were restored to trichothecene production. Isolate GZT201-6 (lane 5) does not contain a copy of the transformation vector.

et al., 1995; VanEtten et al., 1995; Talbot et al., 1993). Although gene disruption is a powerful approach, it has the disadvantage that the transformation process itself can cause alterations in the fungal genome apart from the gene targeted for disruption (Keller et al., 1990; Oliver \& Osbourn, 1995). This phenomenon of nontarget effects of transformation may be particularly problematic when analysing the roles of genes involved in complex phenotypes, such as virulence, where changes at any one of a number of loci could significantly alter the phenotype. In this study, we used gene reversion and mutant complementation to determine whether the reduced virulence of disruption mutants of G. zeae was caused by disruption of Tri5 or by non-target effects of transformation. The gene reversion approach used in this study was based on observations that, during meiosis in other filamentous fungi (i.e. A. nidulans, $G$. pulicaris and Neurospora crassa) homologous recombination between closely linked copies of a duplicated sequence can lead to the loss of one copy of the sequence as well as the sequence between the two copies (Desjardins et al., 1992; Miller et al., 1986; Selker et al., 1987). Disruption mutant GZT33 has two copies of a $1.1 \mathrm{~kb}$ region of the Tri5 coding region separated from each other by approximately $3 \mathrm{~kb}$ of sequence corresponding to a bacterial cloning vector (Fig. 1) (Proctor et al., 1995). We speculated that if this mutant passed through the sexual phase of its life cycle, the duplicated regions might recombine in such a way as to restore the wild-type Tri5 coding region in some progeny. Because G. zeae is homothallic, a single strain of the fungus can complete the sexual phase. We supposed that progeny that resulted from selfing and that had recombined at Tri 5 would be genetically identical to their parent except for the structure of Tri5. Thus, if the reduced virulence of the Tri5 disruption mutant were due to non-target effects of transformation (i.e. an alteration at a locus other than Tri5), revertant progeny should also exhibit reduced virulence. Alternatively, if the reduced virulence were caused by Tri5 disruption, the revertant should exhibit wild-type levels of virulence. The latter alternative appears to be the case, since the trichotheceneproducing, Tri5 revertant progeny, GZP3-9, exhibited wild-type levels of virulence on wheat seedlings. These results are consistent with those from an experiment in which genetically marked (via transformation) derivatives of GZP3-9 exhibited wild-type levels of head blight on wheat in the field (Desjardins et al., 1996).

The interpretation of the Tri5 revertant phenotypes was complicated by progeny GZP1-6 and GZP1-15, in which reversion of the disrupted Tri5 to wild-type was not accompanied by restoration of trichothecene production. The cause of this inability to produce trichothecenes is not known. Initially, we speculated that a nucleotide sequence error may have been introduced into the Tri5 coding region of progeny GZP1-6 and GZP1-15 via the disruption vector, pGZTS4-1 (Proctor et al., 1995). The two rounds of Taq PCR to which the truncated Tri5 coding region in the disruption vector was subjected could have introduced an error in this DNA. The error could have been incorporated into the Tri5 of GZP1-6 and GZP1-15 through recombination events leading to the initial disruption and subsequent reversion of Tri5. That such an error existed was ruled out by nucleotide sequence analysis which revealed that the Tri5 coding regions in the disruption vector, progeny GZP1-6, and strain GZ3639 (wild-type) were identical, except for the expected truncation and $H y g B$ insertion in the disruption vector. We also speculated that the inability of GZP1-6 and GZP1-15 to produce trichothecenes may have been caused by repeated-induced point mutations (RIP) and methylation induced premeiotically (MIP). RIP and/or MIP occur in N. crassa and Ascobolus immersus when strains carrying two or more copies of a gene pass through the sexual cycle and result in the inactivation of all copies of the repeated gene (Cambareri et al., 1989; Rhounim et al., 1992). Nucleotide sequence analysis of the GZP1-6 Tri5 coding region revealed no differences from that of the wild-type (GZ3639) coding region (data not shown). This indicates that a RIP-like phenomenon had not occurred in the trichothecene-deficient revertant progeny. Likewise, Southern blot analysis employing methylation-sensitive and -insensitive enzymes (Sau3AI and $\mathrm{MboI}$ ) and the Tri5 coding region as a hybridization probe, revealed no evidence for a MIP-like phenomenon 
in the trichothecene-deficient revertant progeny (data not shown). The inability of progeny GZP1-6 and GZP1-15 to produce trichothecenes reveals the limitations of the reversion approach, namely revertants may not be isogenic with their parent and revertants may acquire other genetic alterations that affect the phenotype of interest. However, the fact that progeny GZP1-6 and GZP1-15 were unable to produce trichothecenes and exhibited reduced virulence on wheat seedlings strengthens the correlation between trichothecene production and virulence.

That the structure of Tri5 in progeny GZP5-15 is the same as the Tri5-3 allele in disruption mutant GZT40 (Proctor et al., 1995) suggests that the two predicted types of intrachromatid homologous recombination events may have occurred as GZT33 passed through its sexual phase. Intrachromatid recombination between the $5^{\prime}$ ends of the duplicated Tri5 coding regions (i.e. upstream of the $H y g B$ gene insert, Fig. 1) should yield the Tri5-3 allele, while intrachromatid recombination between the $3^{\prime}$ ends of the Tri5 coding regions should yield the Tri5-1 (wild-type) allele. However, the Tri5-1 and Tri5-3 alleles and other constructs could also be generated via crossing over.

The rationale behind the complementation approach was that if the disrupted Tri5 in GZT40 was responsible for the reduced virulence of this mutant, introduction of a functional Tri5 should restore high levels of virulence. However, if the reduced virulence of GZT40 were caused by non-target effects of transformation, introduction of a functional Tri5 would not restore wild-type levels of virulence to the mutant. The fact that the two complemented mutants examined, GZT201-1 and GZT201-5, exhibited relatively high levels of virulence on wheat seedlings indicates that the reduced virulence of GZT40 was due primarily to disruption of Tri5 rather than non-target effects of transformation.

The disadvantage of the complementation approach is that it employs a second transformation step to determine whether an earlier transformation altered the phenotype of interest. This problem was not evident in the current study since the complemented strains were more virulent than the mutant (GZT40) from which they were derived. However, the second transformation, or perhaps the combination of two transformations, may have caused the slightly reduced virulence of strain GZT201-1 as compared to the wild-type progenitor.

Data from both the revertant and complementation approaches provide further evidence that the ability to produce trichothecenes enhances the ability of G. zeae to cause seedling blight. These results combined with results from recent field studies with Tri5 disruption mutants and revertants (Desjardins et al., 1996) support the hypothesis that trichothecenes are virulence factors of G. zeae on both seedlings and adult plants of wheat.

\section{ACKNOWLEDGEMENTS}

We thank A. E. Desjardins for helpful comments throughout this study, K. M. MacDonald and M. L. Moore for excellent technical assistance, L.W. Tjarks for preparing oligonucleotides and running sequencing gels, R. F. Sylvester for preparing figures and T. C. Nelsen for statistical analysis of virulence data.

\section{REFERENCES}

Atanassov, Z., Nakamura, C., Mori, N., Kaneda, C., Kato, H., Jin, Y. Z., Yoshizawa, T. \& Murai, K. (1994). Mycotoxin production and pathogenicity of Fusarium species and wheat resistance to Fusarium head blight. Can J Bot 72, 161-167.

Bowyer, P., Clarke, B. R., Lunness, P., Daniels, M. J. \& Osbourn, A. E. (1995). Host range of a plant pathogenic fungus determined by a saponin detoxifying enzyme. Science 267, 371-374.

Cambareri, E. B., Jensen, B. C., Schabtach, E. \& Selker, E. U. (1989). Repeat-induced G-C to A-T mutations in Neurospora. Science 244, 1571-1575.

Cook, R. J. (1981). Fusarium diseases of wheat and other small grains in North America. In Fusarium: Diseases, Biology and Taxonomy, pp. 39-52. Edited by P. E. Nelson, T. A. Toussoun \& R. J. Cook. University Park, PA: The Pennsylvania State University Press.

Correll, J. C., Klittich, C. J. R. \& Leslie, J. F. (1987). Nitrate nonutilizing mutants of Fusarium oxysporum and their use in vegetative compatibility tests. Phytopathology 77, 1640-1646.

Corrick, C. M. \& Hynes, M. J. (1987). The nucleotide sequence of the amdS gene of Aspergillus nidulans and the molecular characterization of $5^{\prime}$ mutations. Gene 53, 63-71.

Desjardins, A. E., Spencer, G. F., Plattner, R. D. \& Beremand, M. N. (1989). Furanocoumarin phytoalexins, trichothecene toxins, and infection of Pastinaca sativa by Fusarium sporotrichioides. Phytopathology 79, 170-175.

Desjardins, A. E., Hohn, T. M. \& McCormick, S. P. (1992). Effect of gene disruption of trichodiene synthase on the virulence of Gibberella pulicaris. Mol Plant-Microbe Interact 5, 214-222.

Desjardins, A. E., Proctor, R. H., Bai, G., McCormick, S. P., Shaner, G., Buechley, G. \& Hohn, T. M. (1996). Reduced virulence of trichothecene antibiotic-nonproducing mutants of Gibberella zeae in wheat field tests. Mol Plant-Microbe Interact 9, 775-781.

Herrmann, M., Zocher, R. \& Haese, A. (1996). Effect of disruption of the enniatin synthetase gene on the virulence of Fusarium avenaceum. Mol Plant-Microbe Interact 9, 226-232.

Hohn, T. M. \& Desjardins, A. E. (1992). Isolation and gene disruption of the Tox5 gene encoding trichodiene synthase in Gibberella pulicaris. Mol Plant-Microbe Interact 5, 249-256.

Hohn, T. M. \& VanMiddlesworth, F. (1986). Purification and characterization of the sesquiterpene cyclase trichodiene synthetase from Fusarium sporotrichioides. Arch Biochem Biophys 251, 756-761.

Jarvis, B. B. (1991). Macrocyclic trichothecenes. In Mycotoxins and Phytoalexins, pp. 361-421. Edited by R. P. Sharma \& D. K. Salunkhe. Boca Raton, FL: CRC Press.

Jarvis, B. B., Mokhtari-Rejali, N., Schenkel, E. P., Barros, C. S. \& Matzenbacher, N.I. (1991). Trichothecene mycotoxins from Brazilian Baccharis species. Phytochemistry 30, 789-797.

Keller, N. P., Bergstrom, G. C. \& Yoder, O. C. (1990). Effects of genetic transformation on fitness of Cochliobolus heterostrophus. Phytopathology 80, 1166-1173.

Klittich, C. J. R. \& Leslie, J. F. (1988). Nitrate reduction mutants of Fusarium moniliforme (Gibberella fujikuroi). Genetics 118, 417-423.

Kommedahl, T. \& Windels, C. E. (1981). Root-, stalk-, and ear- 
infecting Fusarium species on corn in the USA. In Fusarium: Diseases, Biology and Taxonomy, pp. 94-103. Edited by P. E. Nelson, T. A. Toussoun \& R. J. Cook. University Park, PA: The Pennsylvania State University Press.

Miller, B. L., Miller, K. Y. \& Timberlake, W. E. (1986). Direct and indirect gene replacements in Aspergillus nidulans. Mol Cell Biol 5, 1714-1721.

Nelson, P. E., Pennypacker, B. W., Toussoun, T. A. \& Horst, R. K. (1975). Fusarium stub dieback of carnation. Phytopathology 65, 575-581.

Oliver, R. \& Osbourn, A. (1995). Molecular dissection of fungal phytopathogenicity. Microbiology 141, 1-9.

Panaccione, D. G., Scott-Craig, J. S., Pocard, J. A. \& Walton, J. D. (1992). A cyclic peptide synthetase gene required for pathogenicity of the fungus Cochliobolus carbonum on maize. Proc Natl Acad Sci USA 89, 6590-6594.

Proctor, R. H., Hohn, T. M. \& McCormick, S. P. (1995). Reduced virulence of Gibberella zeae caused by disruption of a trichothecene toxin biosynthectic gene. Mol Plant-Microbe Interact 8, 593-601.

Rhounim, L., Rossignol, J. L. \& Faugeron, G. (1992). Epimutation of repeated genes in Ascobolus immersus. EMBO J 11, 4451-4457.

Rogers, L. M., Flaishman, M. A. \& Kolattukudy, P. E. (1994). Cutinase gene disruption in Fusarium solani f. sp. pisi decreases its virulence on pea. Plant Cell 6, 935-945.

Salch, Y. P. \& Beremand, M. N. (1993). Gibberella pulicaris transformants: state of transforming DNA during asexual and sexual growth. Curr Genet 23, 343-350.

Sambrook, J., Fritsch, E. F. \& Maniatis, T. (1989). Molecular Cloning: A Laboratory Manual. Cold Spring Harbor, NY: Cold Spring Harbor Laboratory.

Selker, E. U., Cambareri, E. B., Jensen, B. C. \& Haack, K. R. (1987).
Rearrangement of duplicated DNA in specialized cells of Neurospora. Cell 51, 741-752.

Sharma, R. P. \& Kim, Y. W. (1991). Trichothecenes. In Mycotoxins and Phytoalexins, pp. 339-359. Edited by R. P. Sharma \& D. K. Salunkhe. Boca Raton, FL: CRC Press.

Stahl, D. J., Theuerkauf, A., Heitefuss, R. \& Schafer, W. (1994). Cutinase of Nectria haematococca (Fusarium solani f. sp. pisi) is not required for fungal virulence or organ specificity on pea. Mol Plant-Microbe Interact 7, 713-725.

Talbot, N. J., Ebbole, D. J. \& Hamer, J. E. (1993). Identification and characterization of MPG1, a gene involved in pathogenicity from the rice blast fungus Magnaporthe grisea. Plant Cell 5, 1575-1590.

Talbot, N. J., Kershaw, M. J., Wakley, G. E., deVries, O. M. H., Wessels, J. G. H. \& Hamer, J. E. (1996). MPG1 encodes a fungal hydrophobin involved in surface interactions during infectionrelated development of Magnaporthe grisea. Plant Cell 8, 985-999.

Tuite, J. (1969). Plant Pathological Methods : Fungi and Bacteria. Minneapolis, MN: Burgess Publishing Company.

Turgeon, B. G., Garber, R. C. \& Yoder, O. C. (1987). Development of a fungal transformation system based on selection of sequences with promoter activity. Mol Cell Biol 7, 3297-3305.

VanEtten, H. D., Sandrock, R. W., Wasmann, C. C., Soby, S. D., McCluskey, K. \& Wang, P. (1995). Detoxification of phytoanticipins and phytoalexins by phytopathogenic fungi. Can J Bot 73 suppl. 1, S518-S525.

Wang, Y.Z. \& Miller, J.D. (1988). Effects of Fusarium graminearum metabolites on wheat tissue in relation to Fusarium head blight resistance. J Phytopathol 122, 118-125.

Received 24 December 1996; revised 7 April 1997; accepted 14 April 1997. 